\title{
Modeling and Stability Analysis of Closed Loop Current-Mode Controlled Cuk Converter using Takagi-Sugeno Fuzzy Approach
}

\author{
Kamyar Mehran, Member IEEE, Damian Giaouris, Member IEEE, and Bashar Zahawi, Senior Member IEEE \\ School of Electrical, Electronic and Computer Engineering, \\ Newcastle University, Newcastle upon Tyne, UK \\ E-mail: \{kamyar.mehran@ncl.ac.uk\}
}

\begin{abstract}
The current-mode controlled Cuk converter, being a fourth-order nonlinear nonsmooth system, does not lend itself to any simple analysis and the complexity lies mainly in the modeling of the circuit to capture all the essential nonlinearities that occur in fast time scale. This paper, for the first time, extends the Takagi-Sugeno fuzzy modeling approach to capture fast time-scale nonlinearities in the converter like border-collision bifurcation and chaos. Then, non-smooth Lyapunov theory is employed to study the stability and actually pinpoint the operating point at which the converter loses its stable period-1 operation to bifurcation.
\end{abstract}

\section{INTRODUCTION}

D C-DC SWITCHING converters are a traditional benchmark for testing different nonlinear modeling and stability analysis approaches, due to their inherent nonsmooth characteristics. Closed loop current-mode controlled Ćuk converter is a fourth-order nonsmooth dynamical system that shows a variety of nonlinear phenomena. Up to this date, the number of modeling methods proposed to capture all the nonlinearities in power electronic converters like period-doubling bifurcation,subharmonics and chaos, although powerful, is limited. Accurate mathematical modeling turns out to be even more essential for power electronic circuits like Ćuk converter. It is mainly because analysis and simulations for the converter, as a fourth-order system, will become rather complicated if we intend to identify all nonlinear phenomena occurring on fast-time scale and not to use the traditional averaged modeling technique, merely capable of characterizing lowfrequency behavior [1]. In exploring new methods for nonlinear modeling of the converter, a novel Takagi-Sugeno fuzzy modeling approach is proposed to study the instabilities in the Ćuk converter, which occurs at clock frequency. Despite other complicated approaches based on discrete nonlinear modeling [1], [2], the proposed TS fuzzy modeling approach seems a promising method to simulate high-frequency behavior of the converter to acceptable accuracy and with low computational time [3].

At the same time, the traditional approach for the stability analysis of the converter is to obtain the period- $n$ equilibrium orbit or limit cycle by setting $x(0)=x(n T)$ acquired from iterated map. Then, the stability of the limit cycle can be determined by checking if the magnitude of all characteristic multipliers at the limit cycle are less than unity [1], [4].
However, using the classical iterative map approach will be ended up with the fourth-order iterative map which makes the stability analysis for higher period-doubling bifurcation fairly knotty. In another attempt, Filippov's method is successfully applied to study the stability of the free-running current controlled Ćuk converter [2]. Even though the method is essentially used for slow-scale stability of the converter, the complexity is inevitable. As an alternative approach , for the first time in this paper, the proposed Takagi-Sugeno model-based system is employed to accurately investigate the stability of the nonsmooth model of the converter and predict the operating point when the converter loses it's stability to border collision bifurcation. The stability analysis will be based on nonsmooth Lyapunov approach which seems realistic [5] for the nonsmooth nature of the model and be formulated numerically as a Linear Matrix inequality (LMI) system. Less computational complexity and more direct analysis by checking the feasibility of the LMI system, take an edge comparing to the former analysis using nonlinear iterative map. Further more, based on LMI stability conditions which will be presented in this paper, a new control strategy can be elaborated to extend the period-1 behavior to the wide range of operating-point excursion. The method has been previously applied to non-autonomous system like boost and buck converter and showed promising results [3], [6].

\section{THE ĆUK CONVERTER AND ITS MATHEMATICAL MODEL}

The closed loop current-mode controlled Ćuk converter (1) is an non-autonomous nonsmooth dynamical system that conventionally is controlled by comparing sum of the inductor current, $i_{L 1}+i_{L 2}$, with a reference current $I_{\text {ref }}$ to generate the OFF driving signal for the switch. The switch $S$ is turned on at the beginning of the cycle $t=n T$ and stay there until $i_{L 1}+i_{L 2}$ reaches the value of $I_{\text {ref }}$, then, the switch $S$ is turned OFF, and stay off until the next cycle begins. The control scheme can be formulated as follows [1]:

$$
I_{r e f}-\left(i_{L 1}+i_{L 2}\right)_{n}=\left[\frac{E}{L_{1}}+\frac{v_{C 2, n}-v_{C 1, n}}{L_{2}}\right] d_{n} T
$$

where subscript $n$ denotes values at $t=n T$. Therefore

$$
d_{n}=\frac{I_{r e f}-\left(i_{L 1}+i_{L 2}\right)_{n}}{\left(\frac{E}{L_{1}}+\frac{v_{C 2, n}-v_{C 1, n}}{L_{2}}\right) T}
$$




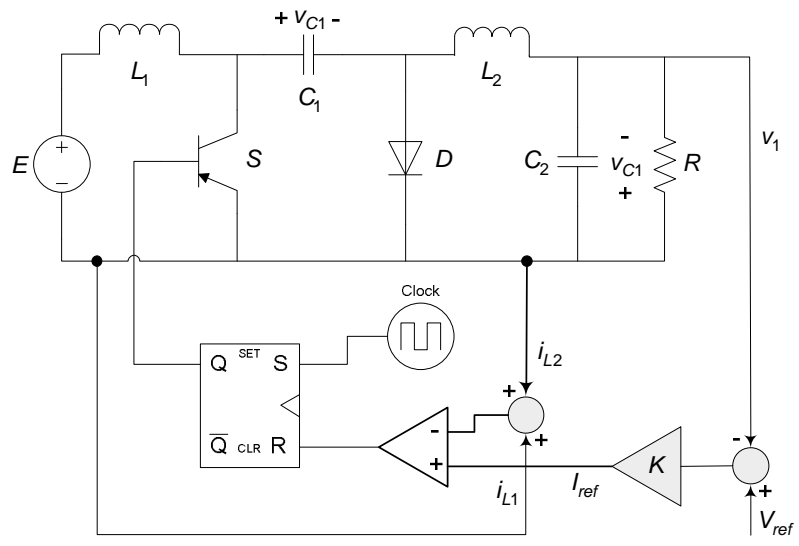

Fig. 1. Ćuk converter under original current-mode control scheme

The dynamic of the system can be described by four sets of differential equations:

$$
\begin{aligned}
& \frac{d v_{C 1}(t)}{d t}= \begin{cases}\frac{-1}{R C 2} v_{C 1}+\frac{1}{C 2} i_{L 1}, & \mathrm{~S} \text { is } \mathrm{OFF} \\
\frac{-1}{R C 2} v_{C 1}+\frac{1}{C 2} i_{L 1}, & \mathrm{~S} \text { is } \mathrm{ON}\end{cases} \\
& \frac{d v_{C 2}(t)}{d t}= \begin{cases}\frac{-1}{C 1} i_{L 1}, \quad \mathrm{~S} \text { is } \mathrm{OFF} \\
\frac{-1}{C 2} i_{L 2}, \quad \mathrm{~S} \text { is } \mathrm{ON}\end{cases} \\
& \frac{d i_{L 1}(t)}{d t}= \begin{cases}\frac{-1}{L 2} v_{C 1}+\frac{1}{L 2} v_{C 2}, & \mathrm{~S} \text { is } \mathrm{OFF} \\
\frac{-1}{L 2} v_{C 1}, & \mathrm{~S} \text { is } \mathrm{ON}\end{cases} \\
& \frac{d i_{L 2}(t)}{d t}= \begin{cases}\frac{1}{L 1} v_{i n}, & \mathrm{~S} \text { is } \mathrm{OFF} \\
\frac{-1}{L 1} v_{C 2}+\frac{1}{L 1} v_{i n}, & \mathrm{~S} \text { is } \mathrm{ON}\end{cases}
\end{aligned}
$$

If we define the state vector as $x=\left[\begin{array}{llll}v_{C 1} & v_{C 2} & i_{L 1} & i_{L 2}\end{array}\right]$ and $u$ as an input voltage $V_{i n}$, the equations (3), (4), (5) and (6) can be written as:

$$
\dot{x}=\left\{\begin{array}{l}
A_{1} x+B u,\left(I_{\text {ref }}-A\left(i_{L 1}+i_{L 2}\right)\right)<i_{\text {ramp }}(t), \\
A_{2} x+B u,\left(I_{\text {ref }}-A\left(i_{L 1}+i_{L 2}\right)\right)>i_{\text {ramp }}(t) .
\end{array}\right.
$$

where:

$$
\begin{array}{r}
A_{1}=\left[\begin{array}{cccc}
-1 / R C 2 & 0 & 1 / C 2 & 0 \\
0 & 0 & -1 / C 1 & 0 \\
-1 / L 2 & 1 / L 2 & 0 & 0 \\
0 & 0 & 0 & 0
\end{array}\right], B=\left[\begin{array}{c}
0 \\
0 \\
0 \\
1 / L 1
\end{array}\right] \text { (7) } \\
A_{2}=\left[\begin{array}{cccc}
-1 / R C 2 & 0 & 1 / C 2 & 0 \\
0 & 0 & 0 & 1 / C 1 \\
-1 / L 2 & 0 & 0 & 0 \\
0 & -1 / L 1 & 0 & 0
\end{array}\right], B=\left[\begin{array}{c}
0 \\
0 \\
0 \\
1 / L 1
\end{array}\right]
\end{array}
$$

In this paper, the proportional feedback controller is used where $i_{\text {ref }}=K\left(V_{\text {ref }}-v_{1}\right), K$ is the control parameter and $V_{\text {ref }}$ is the reference voltage. The outer closed loop determine the reference current $I_{\text {ref }}$ based on the value of $v_{1}$. Under this control scheme the normal output of the converter will be a periodic ripple with the peak value of $I_{\text {ref }}$ and the period that
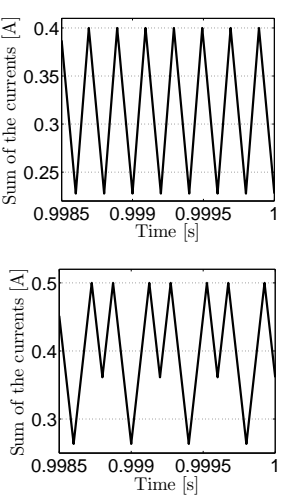

(c)

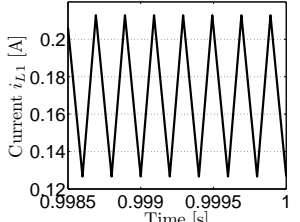

(a)

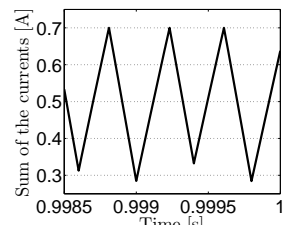

(d) (b)

Fig. 2. The nominal period-1 operation of the Ćuk converter when $I_{\text {ref }}=$ $0.4 A$ for (a) $i_{L 1}+i_{L 2}$ (b) $i_{L 1}$ (c) the output current becomes unstable and behaves in period- 2 operation when $I_{\text {ref }}=0.5 A$ (d) the output current behaves chaotically when $I_{\text {ref }}=0.7 A$. The fixed parameter values are $V_{\text {in }}=$ $15 \mathrm{~V}, L 1=L 2=1 \mathrm{mH}, R=75 \Omega, C=47 \mu F, T=1 / 2500 \mathrm{~s}$.

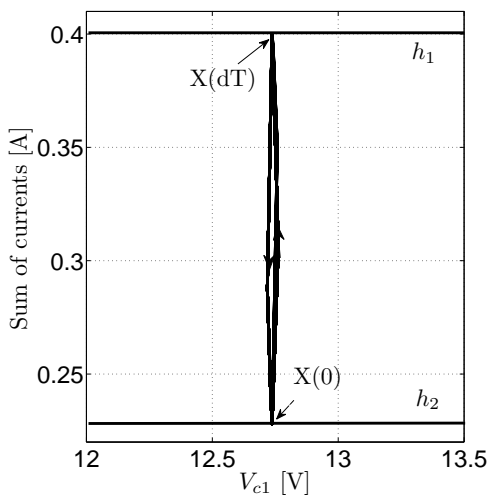

Fig. 3. Period 1 limit cycle:Phase space and the transversal intersection

is equal to the period of the PWM ramp signal. Figure $2 \mathrm{a}$ and $2 \mathrm{~b}$, shows this so-called stable period-1 operation of the converter for sum of the currents $i_{L 1}+i_{L 2}$ and $i_{L 1}$ respectively. Figure $2 \mathrm{c}$ shows the unstable period- 2 operation when border collision bifurcation occurs and Figure 2d illustrates the chaotic behavior under reference-current variation. The example Ćuk converter has two switching manifold on ON and OFF switching instances. Figure 3 illustrates the stable period1 orbit in $v-i$ space, where the orbit periodically switches between $\mathrm{X}(\mathrm{dT})$ and the fixed point of the cycle with Poincare map $\mathrm{X}(0)$. If a system parameter like reference current $I_{\text {ref }}$ is varied, the circuit becomes unstable through period-doubling bifurcation and then chaos, which is apparent from Figure 4 [1].

\section{TS FUZZY MODEL OF THE BUCK CONVERTER FOR FAST-SCALE ANALYSIS}

The fuzzy inference system of Takagi-Sugeno Fuzzy models [7], [8] in order to approximate smooth dynamical functions is generally described by a set of rules in the form

$$
\begin{array}{ll}
\text { Rule } j: & \text { IF } x_{1} \text { is } F_{1}^{j} \text { AND...AND } x_{q} \text { is } F_{q}^{j} \\
& \text { THEN } \dot{x}=A^{j} x+B^{j} u, \quad j=1, \ldots, l
\end{array}
$$




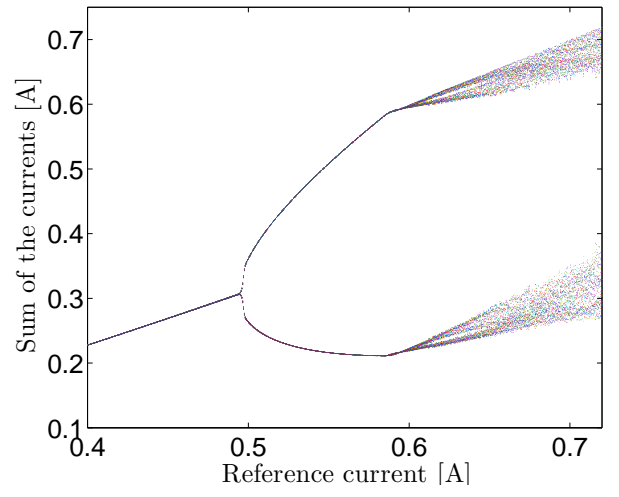

Fig. 4. Bifurcation diagram of the Ćuk converter under closed loop currentmode control (Figure 1), varying reference current as a parameter.

and the dynamics of this system can be described by:

$$
\dot{x}=\sum_{j=1}^{l} w^{j}(x)\left(A^{j} x+B^{j} u\right)
$$

where $w^{j}(x)$ are normalized membership functions of the rule antecedents satisfying $0 \leq w^{j}(x) \leq 1, \sum_{j=1}^{l} w^{j}(\theta)=1$ and $l$ is the number of rules. It is well-known that the model structure above, being famous as affine TS models, is the universal approximator of smooth nonlinear functions to arbitrary accuracy [8], [9]. As discussed in [3], [6], approximating the nonsmooth dynamical function as the example converter with TS fuzzy model structure (9) cannot be realized. The TS model (9) designed to approximate smooth nonlinear functions is fundamentally incapable to represents the inherent discontinuity already observed in the set of equations (3),(4),(5) and (6). In the mathematical point of view, existence (and uniqueness) of the TS fuzzy model of the form (9) is not being hold should the Ćuk converter's non-smooth dynamics modeled with this approach [3], [6]. Nevertheless, deriving the averaged model of the system in Figure 1, makes possible to model the converter with TS modeling approach (9). At the same time, taking advantage of averaging technique to derive an smooth model of the converter, the nonlinearities occurs on fast-time scale will be thoroughly ignored. Therefore, the border-collision bifurcation and chaos occur at the clock frequency [1] is not observable by any means.

The new TS fuzzy modeling approach (11) initially proposed in [3], [6] is capable of representing the essential dynamics of the Ćuk converter (discontinuous), which has the form:

$$
\begin{aligned}
\text { Rule } j & : \operatorname{IF}\left(x_{3}+x_{4}\right) \text { is } F^{j} \\
\text { THEN } \dot{x} & =\left\{\begin{array}{l}
A^{j}\left(m_{i}\right) x+B^{j}\left(m_{i}\right) u \\
m^{+}=\phi(x, m), \quad j=1,2, \quad i=1,2
\end{array}\right.
\end{aligned}
$$

and by the appropriately restricting the inference parameters, the dynamics of the discontinuous fuzzy system can be described by:

$$
\left\{\begin{array}{l}
\dot{x}=\sum_{j=1}^{l} w^{j}\left(x, m_{i}\right)\left(A^{j}\left(m_{i}\right) x+B^{j}\left(m_{i}\right) u\right) \\
m^{+}=\phi(x, m)
\end{array}\right.
$$

where $x \in R^{n}$ is the continuous state, $m \in M=\left\{m_{1}, m_{2}\right\}$ is the discrete state, $A^{j}\left(m_{i}\right) \in \Re^{n \times n}, B^{j}\left(m_{i}\right) \in \Re^{n}$, $w^{j}: \Re^{n} \times M \rightarrow\left[\begin{array}{ll}0 & 1\end{array}\right], j \in I_{l}$, are continuous weighting functions which satisfy $\sum_{j=1}^{l} w^{j}(x, m)=1, l$ is the number of fuzzy rules and $F^{j}$ are fuzzy sets. The state space is the Cartesian product $\Re^{n} \times M$. The function $\phi: \Re^{n} \times M \rightarrow M$ describes the dynamics of the discrete state. The notation $m^{+}$ means the next state of $m$. Any value of discrete state $m_{i} \in M$ is associated with an affine subsystem like:

$$
\text { if } \forall x \in A\left(m_{i}\right) x+B\left(m_{i}\right) u \text { then } m_{i} \in M, i \in\{1,2\}
$$

Remark 1: In general a value of $m_{i}$ could be associated with a subset of subsystem as:

if $\forall x \in\left\{\sum_{j \in\{1,2, \ldots\}} w^{j}\left(x, m_{i}\right)\left(A^{j}\left(m_{i}\right) x+B^{j}\left(m_{i}\right) u\right)\right\}$ then $m_{i} \in\left\{m_{1}, m_{2}, \ldots, m_{N}\right\}$ when $N$ is possibly infinite

As it is inferred from (10) and (11), function $\phi$ formally describes the transition of the discrete states. Each transition involves with jumping from the subset of fuzzy subsystems, which from now on is called fuzzy sub-vector field, to another fuzzy sub-vector field. Alternatively, the transition can be defined by a set of switch sets used to mathematically define the switching manifold. The general formulation of the switch sets is defined as:

$$
S_{i, k}=\left\{x \in R^{n} \mid m_{k}=\phi\left(x, m_{i}\right)\right\}, m_{i} \neq m_{k}, i, k \in I_{N}
$$

which in the case of the Cuk converter, the switching hypersurface at the switching OFF

$$
h(X,(d T))=x_{3}(d T)+x_{4}(d T)-K\left(V_{\text {ref }}-x_{1}\right)
$$

in the form of switch sets can be describe as:

$$
\begin{aligned}
& S_{1,2}=\left\{x \in R^{n} \mid x_{3}(d T)+x_{4}(d T)+K x_{1}<K V_{\text {ref }}\right\}, \\
& \left.S_{2,1}=\left\{x \in R^{n} \mid x_{3}(d T)+x_{4}(d T)+K x_{1}>K V_{\text {ref }}\right\}\right\}
\end{aligned}
$$

where $d$ is the duty ratio at each instant and $T$ is the switching period.

To construct the TS model (10) of the converter, the membership functions are defined to exactly represent each fuzzy sub-vector as follows:

$$
\begin{aligned}
F^{1}\left(x_{3}(t)+x_{4}(t)\right) & =\frac{1}{2}\left(1+\frac{X_{1}(0)-x_{3}(t)-x_{4}(t)}{2 l}\right), \\
F^{2}\left(x_{3}(t)+x_{4}(t)\right) & =\frac{1}{2}\left(1+\frac{X_{1}(0)-x_{3}(t)-x_{4}(t)}{2 l}\right)
\end{aligned}
$$

Here, $l$ is a constant denoting the range of sum of the currents, i.e. $i_{L 1}(t)+i_{L 2}(t) \in\{0.4-l, 0.4+l\}$. We let $l=0.2$. The state vector $X(0)=\left[\begin{array}{llll}12.7187 & 27.5608 & 0.1012 & 0.1268\end{array}\right]$ is the transversal intersection of the periodic orbit with the switching manifold when the switch is OFF (see Figure 3). Normally we would pick the equilibrium point of the system if destined for modeling a smooth or the average model of the converter [10]. However, the nonsmooth model is the target here so the transversal intersection point should take into consideration in the membership function to accurately represents switching between two sub-vector fields. Since the linear vector fields are dealt with in this case, the matrices in (7) and (8) can immediately be used in building the exact TS fuzzy models of the converter as $A^{1}\left(m_{1}\right)=A^{2}\left(m_{1}\right)=A_{1}$, $A^{1}\left(m_{2}\right)=A^{2}\left(m_{2}\right)=A_{2}$ and $B\left(m_{1}\right)=B\left(m_{2}\right)=B$ where the discrete states $m_{1}$ and $m_{2}$ is actually representing $\mathrm{ON}$ 
and OFF state of the converter respectively. Figure 5a and $5 \mathrm{~b}$ comparing with the original behavior of the converter in different operation modes (Figure 2) shows that the proposed TS fuzzy model under current-mode control can well represents the nonsmooth switching and capture any phenomena occurring at the clock cycle like border collision bifurcation and chaos. The bifurcation diagram in Figure 6 makes it more evident the strength of the new TS fuzzy modeling method in capturing all nonlinearities by showing exactly the same quantitative behavior compared with Figure 4.

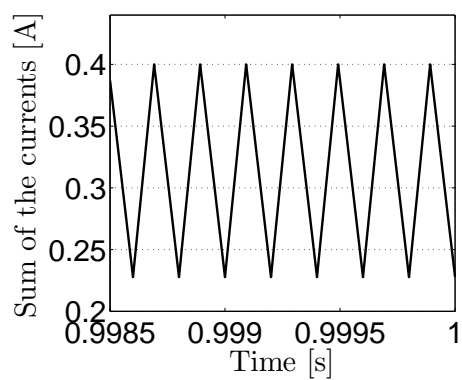

(a)

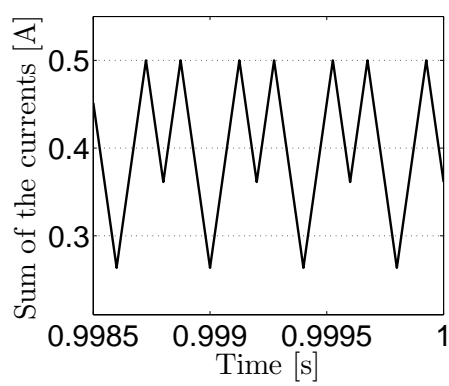

Fig. 5. (a) The nominal period-1 operation of the TS fuzzy model of the Ćuk converter under original current-mode control when $I_{\text {ref }}=0.4 \mathrm{~A}$ (b) The nominal period- 2 operation of the TS fuzzy model of the converter when $I_{\text {ref }}=0.5 A$

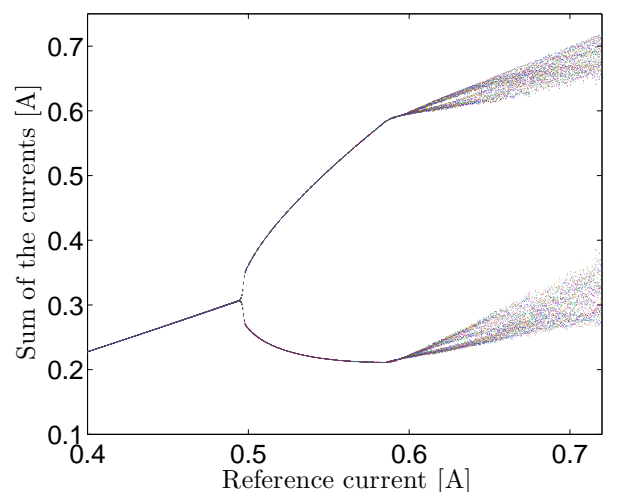

Fig. 6. Bifurcation diagram of the Ćuk converter modeled with TS fuzzy approach, varying reference current as a parameter.

\section{EXPONENTIAL STABILITY ANALYSIS}

The stability of the limit cycle in power electronics circuits will be predominately determined using Poincaré maps [11]. At a selected switching surface, a perturbation of the switching point is mapped through the switching cycle back to the same switching surface. This is a discrete mapping, where the eigenvalues of the mapping matrix determines the stability of the limit cycle. Employing this approach with an engagement of Filippov's method, the stability analysis of an autonomous Ćuk converter is already studied [2].

\section{A. Nonsmooth Lyapunov method to show the stability of limit cycle}

Considering the fact that the proposed TS fuzzy model of the converter represents a nonsmooth dynamical system,a Lyapunov method is employed for the first time in this section for the bifurcation analysis of the example converter. The traditional approach of finding a smooth global Lyapunov function to show the stability of TS fuzzy models approximating a smooth nonlinear system have been around for long time in the model-based fuzzy control literature [8]. The shortcomings of finding global quadratic Lyapunov candidates in the entire fuzzy state space and the subsequent conservative LMI formulation for TS fuzzy models of the smooth nonlinear systems and naturally for the proposed TS fuzzy model (11) is also already hashed over [3], [6], [12]. Briefly, to relax the Linear Matrix Inequalities (LMI) formulation for the stability analysis of the nonsmooth model of the converter, the Lyapunov function candidate should be given as discontinuous or nonsmooth functions. We also let the fuzzy state space to be partitioned to different flexible regions. A switching function partitions the state space into two regions, separated by the switching surface $h(X,(d T))=0$. Followingly, we let the fuzzy state space $\mathcal{F}$ is partitioned into two detached region $\Omega_{q}, q \in I_{2}$ where $I_{2}=\{1,2\}$. Therefore

$$
\begin{aligned}
& \Omega_{1}=\left\{(x, m) \in \mathcal{F} \mid x \in \Re^{n}, m=m_{1}\right\} \\
& \Omega_{2}=\left\{(x, m) \in \mathcal{F} \mid x \in \Re^{n}, m=m_{2}\right\}
\end{aligned}
$$

A trajectory initiated in any region at time $t_{k}, k=1,2, \ldots$ can pass through another region if $t_{k}<t_{k+1}$. We define $\Lambda_{q r}$ as a neighboring region which means:

$$
\Lambda_{q r}=\left\{x \in \Re^{n} \mid \exists t<t_{0}, \text { such that } x\left(t^{-}\right) \in \Omega_{q}, x(t) \in \Omega_{r}\right\}
$$

$\Lambda_{q r}$ is given by the hypersurface of the form (13). In fact, conditions for the switching from mode $m_{1}$ to mode $m_{2}$ at time $\tau$ are

$$
\left\{\begin{array}{l}
h(X, \tau)=0 \\
\sum_{j \in\{1,2, \ldots\}} w^{j}\left(x, m_{i}\right)\left(A^{j}\left(m_{i}\right) x+B^{j}\left(m_{i}\right) u\right) \cdot \nabla h(X, \tau)<0
\end{array}\right.
$$

Hence if $\Lambda_{q r} \neq \emptyset, \Omega_{q}$ and $\Omega_{r}$ must be neighboring sets. As a sufficient condition let:

$$
\mathrm{I}_{\Lambda}=\left\{(q, r) \mid \Lambda_{q r} \neq \emptyset\right\}
$$

which is a set of tuples indicating that there is at least one point for which the trajectory passes from $\Omega_{q}$ to $\Omega_{r}$.

Now, we define a local quadratic Lyapunov function for each region, which has the structure:

$$
V(x)=V_{q}(x)=\tilde{x}^{T} \tilde{P}_{q} \tilde{x} \text { when }(x, m) \in \Omega_{q}
$$

where $\tilde{x}=\left[\begin{array}{l}x \\ 1\end{array}\right], \tilde{P}_{q}=\left[\begin{array}{cc}P_{q} & p_{q} \\ p_{q}^{T} & \pi_{q}\end{array}\right], \pi_{q} \in \Re, p_{q} \in \Re^{n}, P_{q}=$ $P_{q}^{T} \in \Re^{n} \times \Re^{n}$ and $q \in I_{2}$. 
Let $\Omega_{q}^{x}$ denote the continuous state of $x$ in $\Omega_{q} . V_{q}$ : $\mathbf{c l} \Omega_{q}^{x} \rightarrow \Re, q \in I_{\Delta}$, is a (scalar) function which is assumed to be continuously differentiable on closure of region $\Omega_{q}$ (cl. denotes the closure of a set, which is the smallest closed set containing the set). In fact, the scalar function $V_{q}(x, t)$ is used to measure the fuzzy system's energy in a local region $\Omega_{q}$. As it is apparent, the overall Lyapunov function $V$ (19) is a discontinuous Lyapunov function at the hypersurface (13) or at the neighboring regions $\Lambda_{q r},(q, r) \in I_{\Lambda}$. Assuming $t_{k}<t_{k+1}$ for every trajectory with initial point in any region, $V(x)$ is piecewise continuous function with respect to time.

\section{B. LMI formulation for stability and bifurcation analysis}

The first stage in formulating the Lyapunov stability conditions into LMI conditions is to define fuzzy state-space partitions by (positive) quadratic functions. This is realizable using the so-called S-procedure technique to substitute the confined conditions with unconfined conditions [13]. To explain the procedure in general terms and more clearly, let $Q_{0}, \ldots, Q_{s}$, be quadratic functions of the variable $x \in \Re^{n}$ on the form:

$$
Q_{k}(x)=x^{T} Z_{k} x+2 c_{k}^{T}+d_{k}, k=0, \ldots, s,
$$

where $Z_{k}=Z_{k}^{T}$. We consider the following condition on $Q_{0}$ :

$$
Q_{0}(x) \geq 0 \text { in the region }\left\{x \in \Re^{n} \mid F_{k}(x) \geq 0, k \in I_{s}\right\}
$$

The confined condition (21) can be substituted by an unconfined condition in the following way:

Lemma [13]: if there exist $\delta_{k} \geq 0, k \in I_{s}$, such that

$$
\forall x \in \Re^{n}, Q_{0}(x) \geq \sum_{k=1}^{s} \delta_{k} Q_{k}(x)
$$

then (21) holds. Hence, by introducing additional variables $\delta_{k} \geq 0, k \in I_{s}$, condition (21) can be turned into an LMI which can be written as:

$$
x^{T}\left[\begin{array}{cc}
Z_{0} & c_{0} \\
c_{0}^{T} & d_{0}
\end{array}\right] x \geq \sum_{k=1}^{s} \delta_{k} x^{T}\left[\begin{array}{cc}
Z_{0} & c_{0} \\
c_{0}^{T} & d_{0}
\end{array}\right] x
$$

The replacement of (21) by Lemma may be conservative. However it can be shown that the converse is true in case of single quadratic form, $s=1$ [13] contingent on the existence of some $x$ such that $Q_{1}(x)>0$. In case of hypersurface which can be defined by $Q_{k}(x)=0, k \in I_{s}$, Lemma is true without the restriction $\delta_{k} \geq 0$.

Here, all the conditions in the stability theorem are immediately described by $Q_{0}(x) \geq 0$, where $Q_{0}(x)$ is a quadratic function defined by (20).

$$
\left\{\begin{array}{l}
Q_{0}(x)=\tilde{x}^{T}\left(\tilde{P}_{q}-\alpha \tilde{I}\right) \tilde{x} \geq 0, \forall x \in \Omega_{q}^{x}, q \in I_{2} \\
Q_{0}(x)=\tilde{x}^{T}\left(\beta \tilde{I}-\tilde{P}_{q}\right) \tilde{x} \geq 0, \forall x \in \Omega_{q}^{x}, q \in I_{2} \\
Q_{0}(x)=-\tilde{x}^{T}\left(\tilde{A}(m)^{T} \tilde{P}_{q}+\tilde{P}_{q} \tilde{A}(m)+\gamma \tilde{I}\right) \tilde{x} \geq 0, \forall(x, m) \\
Q_{0}(x)=\tilde{x}^{T}\left(\tilde{P}_{r}-\tilde{P}_{q}\right) \tilde{x} \geq 0, \forall x \in \Lambda_{q r}^{x}, \quad(q, r) \in \mathrm{I}_{\Lambda}
\end{array}\right.
$$

In the conditions above, $\alpha$ and $\beta$ are constants which originally represent class $\mathcal{K}$ function $\alpha(\|x\|), \alpha: \Re^{+} \rightarrow \Re^{+}$and $\beta(\|x\|)$, $\beta: \Re^{+} \rightarrow \Re^{+}$.(for definition of class $\mathcal{K}$ function see [14]) and $\gamma>0$ is a scalar constant. The third condition is satisfied on the hypersurface $\Lambda_{q r}^{x}$ which can be given by $Q_{k}(x)=$
$0, k \in I_{s}$, where each $Q_{k}(x)=0$ has the form (20) with no limitation on $\delta_{k}$ as mentioned before. All the conditions can be substituted by the unconfined condition (23); however, if the switching manifold cannot exactly be represented by $Q_{k}(x)=$ $0, k \in I_{s}$ in the last condition, it is possible to represent such a region with quadratic fucntions satisfying $Q_{k}(x) \geq 0$, in which the additional variables $\delta_{k}$ should be limited to $\delta_{k} \geq 0$.

Now, all the stability conditions for bifurcation analysis can be recasted to LMI conditions:

LMI problem: If there exist $\tilde{P}_{q}, q \in I_{\Delta}$, constants $\alpha>0$, $\mu_{k}^{q} \geq 0, \nu_{k}^{q i j} \geq 0, \eta_{k}^{q r}$ and a solution to $\min \beta$ subject to the three conditions:

$$
\begin{gathered}
-\alpha \tilde{I}+\sum_{k=1}^{s_{q}} \mu_{k}^{q}\left[\begin{array}{cc}
Z_{k}^{q} & c_{k}^{q} \\
\left(c_{k}^{q}\right)^{T} & d_{k}^{q}
\end{array}\right] \leq \tilde{P}_{q} \\
\tilde{P}_{q} \leq \beta \tilde{I}+\sum_{k=1}^{s_{q}} \mu_{k}^{q}\left[\begin{array}{cc}
Z_{k}^{q} & c_{k}^{q} \\
\left(c_{k}^{q}\right)^{T} & d_{k}^{q}
\end{array}\right], q \in I_{\Delta} \\
\text { - } \quad(q, i, j) \in I_{\Omega},\left(\tilde{A}^{j}\right)^{T} \tilde{P}_{q}+\tilde{P}_{q} \tilde{A}^{j} \\
+\sum_{k=1}^{s_{q i j}} \nu_{k}^{q i j}\left[\begin{array}{cc}
Z_{k}^{q} & c_{k}^{q} \\
\left(c_{k}^{q}\right)^{T} & d_{k}^{q}
\end{array}\right] \leq-\tilde{I}, q \in I_{\Delta} \\
\text { - } \tilde{P}_{r} \leq \tilde{P}_{q}-\sum_{k=1}^{s_{q r}} \eta_{k}^{q r}\left[\begin{array}{cc}
Z_{k}^{q r} & c_{k}^{q r} \\
\left(c_{k}^{q r}\right)^{T} & d_{k}^{q r}
\end{array}\right],(q, r) \in I_{\Lambda}
\end{gathered}
$$

Then the fixed point 0 is exponentially stable in the sense of Lyapunov ${ }^{1}$.

Remark 2: Without loss of generality, it is assumed that the origin is a fixed point of the fuzzy system (11). For the buck converter, the fixed point mentioned above is the fixed point of limit cycle with a stroboscopic map [15] $\square$

Let the fixed parameter values as mentioned in Figure 2 , for a specified desired current $I_{\text {ref }}=0.42$, which is the stable period-1 operation of the converter as apparent from the bifurcation diagram in Figure 6, the system converges exponentially to the stable limit cycle, which will be verified by solving LMI problem.

$$
\tilde{\mathbf{P}}_{\mathbf{1}}=\left[\begin{array}{ccccc}
43.8488 & 41.3433 & -93.4600 & 0 & 0 \\
41.3433 & 42.5052 & -90.0070 & 0 & 0 \\
-93.46 & -90.00 & 810.9474 & 0 & 0 \\
0 & 0 & 0 & 1.33 & -833.36 \\
0 & 0 & 0 & -833.36 & 2.08 \\
& & & & (25)
\end{array}\right]
$$

$$
\in \tilde{\mathbf{P}}_{\mathbf{2}}=\left[\begin{array}{ccccc}
1.0210 & 0 & -1.8009 & 0 & 0 \\
0 & 1.6300 & 0 & -0.0050 & -24.44 \\
-1.8009 & 0 & 269.87 & 0 & 0 \\
0 & -0.0050 & 0 & 554.86 & -0.14 \\
0 & -24.44 & 0 & -0.147 & 949.35 \\
& & & & (26)
\end{array}\right.
$$

with the optimal value of $\alpha=0.36$ and $\beta=0.43$. If the value of reference current is intentionally changed to $I_{\text {ref }}=0.5$, the LMI problem founds infeasible and no optimal value of $\alpha$ and

\footnotetext{
${ }^{1}$ The proof of this theorem is out of the scope of this paper and it will present in later publications.
} 
$\beta$ can be found. Actually, the infeasibility of LMI problem, can detect the edge of unstable period doubling bifurcation as for all the values of $I_{\text {ref }}<0.5$, the LMI problem can found a feasible solution with the optimum value of $\beta$. This readily determine the range of the stable period-1 operation of the converter without using nonlinear discrete mapping approach.

Letting the fuzzy state space to be partitioned into flexible detached region is of prime importance in finding the actual stability of the nonsmooth system like the example Cuk converter. An attempt to let the overall Lyapunov function $V$ measure the system's energy for the entire fuzzy state space means a common Lyapunov function for all discrete states has to be sought. Then, the neighboring region $\Lambda_{q r}$ are all empty and and the system will be stable regardless of the switchings. However, as can be noticed from table below, searching for the feasible solution to the LMI problem is not possible even for the stable period- 1 excursion of the converter. Partitioning the fuzzy state space into more regions may end up in different optimal value of $\beta$, although it can accurately show the stable region of the converter and the operating point when bifurcation occurs by the LMI feasibility problem. Deciding a suitable region partitioning is optional. Nevertheless, it should be avoided to make numerous partitions or a partitioning so complex as in practice, verifying the stability conditions in the LMI problem turns out to be impossible for heavy computational burden.

\begin{tabular}{c|c|c|c}
$\begin{array}{c}\text { Number of } \\
\text { Partitions in } \mathcal{F}\end{array}$ & $\begin{array}{c}\text { LMI } \\
\text { feasibility }\end{array}$ & $\begin{array}{c}\text { Optimum } \\
\text { value of } \beta\end{array}$ & $\begin{array}{c}\text { Numerical } \\
\text { complexity }\end{array}$ \\
\hline \hline 1 & not feasible & N/A & low \\
\hline 2 & feasible & 0.4330 & low \\
\hline 4 & feasible & 0.4540 & medium \\
\hline 8 & feasible & 0.4540 & medium \\
\hline 16 & feasible & 0.6450 & high \\
\hline 32 & not feasible & N/A & very high
\end{tabular}

\section{CONCLUSION}

The Takagi-Sugeno fuzzy modeling approach proposed in this paper is specifically synthesized to incorporate the typical switching events of power electronic converters. Application on the example Ćuk converter, showed the prowess of new TS fuzzy modeling approach to capture all nonlinear phenomena occurs at the clock cycle like fast-scale bifurcation and chaos.

A rigorous mathematical stability analysis based on the piecewise Lyapunov function candidates introduced to delve into the stability of limit cycles. All stability conditions formulated in Linear Matrix Inequalities (LMI) conditions to evidently predict the onset of unstable period-doubling bifurcation. Presented nonsmooth Lyapunov theory seems essential to tackle the discontinuous dynamics of the new TS fuzzy model of the converter. Fuzzy state-space partitioning to relaxed, detached regions reduce the possibility of conservative LMI formulation to bare minimum although a suitable region partitioning is ad-hoc to each case. The proposed stability analysis is a new way to get an insight of all the fast-scale instabilities of the converter over and above the traditional discrete nonlinear mapping normally employed for specially high-ordered systems.
This paper demonstrates another successful attempt of the proposed modeling and stability analysis approach on power electronic circuits. Not only the whole approach can be extended to tackle the nonsmooth mechanical systems with state jumps, it is also a solid bedrock to coin new switching fuzzy model-based controllers to preserve the stable period-1 behavior of the power electronic circuits for large range of parameter variation.

\section{REFERENCES}

[1] C. K. Tse, Complex Behavior of Switching Power Converters. CRC Press, 2004

[2] I. Daho, D. Giaouris, B. Zahawi, V. Picker, and S. Banerjee, "Stability analysis and bifurcation control of hysteresis current controlled cuk converter using Filippovs method," in Power Electronics, Machines and Drives, 2008. PEMD 2008. 4th IET Conference on, pp. 381-385, 2008.

[3] K. Mehran, D. Giaouris, and B. Zahawi, "Modeling and stability analysis of dc-dc buck converter via takagi-sugeno fuzzy approach," in IEEE Conference on Intelligent Systems and Knowledge Engineering, (Xiamen, China), IEEE, November 2008.

[4] C. Tse and W. Chan, "Instability and chaos in a current-mode controlled Cuk converter," in Power Electronics Specialists Conference, 1995. PESC'95 Record., 26th Annual IEEE, vol. 1, 1995.

[5] D. Shevits and B. Paden, "Lyapunov stability theory of nonsmooth systems," IEEE Transactions on Automatic Control, vol. 39, no. 9, pp. 1910-1914, 1994.

[6] K. Mehran, D. Giaouris, and B. Zahawi, "Stability analysis and control of nonlinear phenamena in boost converter using model-based takagisugeno fuzzy approach," submitted to Circuits and Systems - I, 2008.

[7] T. Takagi and M. Sugeno, "Fuzzy identification of systems and its applications to modeling and control," IEEE Transaction on Systems, Man and Cybernetics, vol. 15, no. 1, pp. 116-132, 1985.

[8] K. Tanaka, Fuzzy Control Systems Design and Analysis: a Linear Matrix Inequality Approach. Newark: John Wiley \& Sons, 2001.

[9] P. Bergsten, Observers and Controllers for Takagi-Sugeno Fuzzy systems. PhD thesis, Örebro, 2001.

[10] K. Lian, J. Liou, and C. Huang, "LMI-based integral fuzzy control of DC-DC converters," IEEE Transactions of Fuzzy Systems, vol. 14, pp. 71-80, February 2006.

[11] S. Banerjee and G. Verghese, eds., Nonlinear Phenomena in Power Electronics: attractors, bifurcation, chaos, and nonlinear control. New York, NY 10016-5997: IEEE Press, 1 ed., 2001.

[12] M. Johansson, A. Rantzer, and K. Årzén, "Piecewise quadratic stability of fuzzy systems," IEEE Transaction on Fuzzy Systems, vol. 7, no. 6, 1999.

[13] S. Boyd, L. E. Ghaoui, E. Feron, and V. Balakrishnan, Linear Matrix Inequalities in System and Control Theory. Philadelphia: SIAM, 1994.

[14] H. K. Khalil, Nonlinear Systems. Upper Saddle River, New Jersey: Prentice-Hall, 2nd ed., 1996.

[15] D. Giaouris, S. Banerjee, B. Zahawi, and V. Pickert, "Stability analysis of the continuous-conduction-mode buck converter via filippov's method," IEEE Transactions on Circuits and Systems-I, vol. 55, pp. 1084-1096, May 2008. 